\title{
Linx
}

Revue des linguistes de l'université Paris X Nanterre

$12 \mid 2002$

«Comme la lettre dit la vie »

\section{Le complément de nom de parenté, de possession et de dépendance dans Perceval ou le conte du Graal}

Fidèle Diedhiou

\section{(2) OpenEdition}

\section{Journals}

Édition électronique

URL : http://journals.openedition.org/linx/1280

DOI : $10.4000 / \operatorname{linx} .1280$

ISSN : 2118-9692

Éditeur

Presses universitaires de Paris Nanterre

\section{Édition imprimée}

Date de publication : 1 octobre 2002

Pagination : 71-79

ISSN : 0246-8743

\section{Référence électronique}

Fidèle Diedhiou, «Le complément de nom de parenté, de possession et de dépendance dans Perceval ou le conte du Graal », Linx [En ligne], 12 | 2002, mis en ligne le 10 octobre 2012, consulté le 01 mai 2019. URL : http://journals.openedition.org/linx/1280 ; DOI : 10.4000/linx.1280

Ce document a été généré automatiquement le 1 mai 2019.

Département de Sciences du langage, Université Paris Ouest 


\section{Le complément de nom de parenté, de possession et de dépendance ${ }^{1}$ dans Perceval ou le conte du Graal}

Fidèle Diedhiou

\section{Détermination des facteurs commandant l'apparition des différentes tournures.}

\section{A. La tournure sans préposition}

1 Cette tournure a un domaine propre d'apparition qui dépend d'un nombre considérable de facteurs qui ont été presque unanimement reconnus par les grammairiens. ${ }^{3}$ Ces facteurs sont :

I) La sous-catégorisation du complément

2 Pour qu'il y ait la tournure absolue, le complément de nom doit désigner un animé humain appartenant à un rang social élevé. Cela revient à dire que le complément renvoie généralement à une personne de haut rang (roi, reine, prud'homme, chevalier, comte, duc, etc.) :

Fil estes le roy Urïen,

Et avés non mesire Yvains (Yvain, 1016-1017)

Vous êtes le fils du roi Urien et vous vous appelez monseigneur Yvain.

Une fois a le court le roi

M'envoia me dame en message (Yvain, 1002-1003)

Une fois à la cour du roi ma dame m'envoya comme messagère.

2) Le choix d'un déterminant au singulier propre à déterminer de façon précise le complément en le particularisant. 
- Lorsque le complément est un nom propre, il n'est pas précédé d'un déterminant. Il en est ainsi lorsque le complément est Dieu :

Et la roÿne maintenant

Les nouveles Calogrenant

Li reconte tout mot a mot (Yvain, 655-657)

Et la reine tout aussitôt lui répéta mot à mot l'histoire de Calogrenant.

- Lorsque le complément est un nom commun, déjà actualisé par le contexte (un nom déjà cité ), il est accompagné de l'article défini le ou la ${ }^{4}$ :

An son lit trueve Kex dormant

Et puis vint au lit la reïne (La Charrette, 4650-4651)Il trouve Keu endormi dans son lit et s'approcha du lit de la reine.

- Le complément nom commun peut aussi être précédé du possessif :

La fille ma mere:

la fille de ma mère

Soulignons qu'on n'emploie pas la tournure directe lorsque le complément est précédé de l'article indéfini un. Le complément doit désigner une personne bien déterminée au singulier. Il doit être un nom propre, un nom commun ou un pronom.

- Les pronoms susceptibles de se substituer à un nom déterminé de façon précise doivent être normalement des pronoms bien définis, en l'occurrence un pronom démonstratif suivi d'une relative ou un pronom personnel :

Cil...

en est alez sanz demorance

a l'ostel celui qui vaillance

avoit en son cuer. (Le Vair Palefroi, 734-737)

3 Mais, s'il nous arrive souvent de rencontrer la tournure directe avec un complément qui est un pronom démonstratif suivi d'une relative, par contre, phénomène assez étrange, il est très rare et même très exceptionnel de trouver la tournure directe quand le complément est un pronom personnel. En revanche, on rencontre souvent la tournure directe avec un terme aussi indéterminé que le pronom indéfini autrui (cas régime tonique de autre) :

L'autrui joie (Lanval, 257).

La joie d'autrui 
3) Le complément doit avoir une valeur "subjective" et non une valeur "objective". Le complément a une valeur « subjective » lorsqu'il possède ou produit la chose exprimée par le terme complété ou fait l'action énoncée par ce terme. Par contre, le complément a une valeur «objective » lorsqu'il désigne la personne qui est l'objet d'un sentiment. L'expression du français moderne "l'amour de Dieu » est susceptible de deux interprétations différentes : cela peut être l'amour que Dieu éprouve pour ses créatures, Dieu est alors un complément « subjectif ». Cette expression peut aussi bien signifier l'amour dont Dieu est l'objet. Dieu est alors un complément à valeur « objective ». Cette ambiguïté était déjà notable en latin.

En latin, en effet, rien dans la terminaison des substantifs n'indique le sens actif ou le sens passif: Amor Dei pouvait être l'amour que Dieu éprouve tout aussi bien l'amour dont Dieu est l'objet.

L'ancien français Amor Deu (construction directe) constitue un gain de clarté par rapport au latin et au français moderne car il ne peut signifier que l'amour éprouvé par Dieu alors que l'amour dont Dieu est l'objet se dit en ancien français Amor de Deu.

4) Le complément a généralement un contenu sémantique qui exprime soit un lien de parenté (pere, mere, fils, feme, niés, etc.),

Li niés Marsile, il ad num Aëlroth

Le neveu de Marsile s'appelle Aelroth (Ch. de Roland, v. 1188)

soit une condition sociale élevée (roi, reine, comte, seigneur, chevalier, etc.) et notons qu'il y a des survivances en français moderne de cette construction dans la toponymie: Vic-le-Comte, Bourg-la-Reine, Pont-l'Evêque, Bois-le-Roi, La Ferté-Bernard, etc.

\section{B. La tournure avec la préposition a}

1) On emploie a quand le complément n'est pas un nom de personne :

Le cri au chien (Beroul,1530) : le cri du chien

Ici la préposition $a$ indique l'appartenance. Le cri n'est pas celui d'un autre animal.

2) On l'emploie avec des noms désignant une personne :

a) quand ce nom est au pluriel,

b) quand il est indéterminé :

C'une maison a i. hermite

Trouva, mout basse et molt petite. (Yvain, 2829-2830)

Il trouva la maison d'un ermite, qui était très basse et très petite

Nous sentons l'indétermination du complément à travers l'article indéfini un.

c) Quand il désigne une personne de rang social non élevé :

Fieus a putain (Jeu de Saint Nicolas, 134) : fils de putain Filz au vavasor (Charrette, 2177) : fils du vavasseur

d) On peut trouver la construction avec la préposition $a$ là où la règle permettait l'emploi de la simple juxtaposition, comme nous l'avons montré plus haut. 
La fille au seignor (Yvain, 5407) : la fille du seigneur

...et quant il fu hore de mengier, il issirent de la chapele et s'asistrent en la meson au preudome (Queste, 139,12):

Quand il fut temps de manger, ils sortirent de la chapelle et vinrent s'asseoir dans la maison de l'ermite

Nous nous trouvons dans une situation où nous n'avons pas le choix entre les deux constructions. La deuxième se présente comme s'étant imposée.

\section{La tournure avec la préposition de}

1) On la rencontre souvent quand le complément est un nom de chose ou d'animal.

Le cri du chien (Béroul, 1600) : le cri du chien

Il en apele la gent de sa contree (La Prise d'Orange, 301)

Il interpelle les gens de sa région

2) On le rencontre toujours quand le complément est un nom géographique :

Tuit ont chanté de la cité de Nyme: Tous ont chanté la cité de Nîmes

3) On le voit aussi quand il désigne un nom de personne :

a) Lorsque celui-ci est au pluriel :

De trestuz reis vos present les curunes (Roland, 388): Je vous donne en présent les couronnes de tous les rois.

b) Lorsque le complément a une valeur « objective » :

La pitié de son seignor (Béroul, 1478) : La pitié dont le seigneur est l'objet.

Cependant on peut rencontrer des cas où la préposition de est employée là où l'on s'attendrait à la tournure non prépositionnelle. C'est ainsi qu'on peut lire dans la même page 220 de la Queste les deux emplois différents :

...contre le grant engin sa fame (ligne 14)... contre l'engin de sa fame (ligne 21).

On remarque que les deux prépositions ont souvent le même domaine d'emploi. Mais il y a toutefois une spécialisation de la préposition de dans les valeurs d'origine, de matière, de propos et de partie. De plus, on remarque que les deux prépositions viennent, comme le précise G. Moignet (1984: 95) « directement concurrencer dans son domaine d'emploi propre le tour non prépositionnel.»

Après avoir donné la théorie de l'emploi des différentes tournures, nous allons examiner l'application de cette théorie dans le Conte du Graal ou Perceval, œuvre de 9234 vers de la fin du XII siècle ${ }^{5}$. Ce texte nous paraît intéressant pour notre étude syntaxique car il aurait l'avantage de rendre compte non seulement de la syntaxe propre à l'ancien français, mais encore de nous permettre de déceler le moment où apparaissent les tournures qui seront celles du français moderne.

\section{L'emploi des trois tournures dans Perceval ou Le Conte du Graal6}

18 Nous avons recensé 45 emplois de la tournure par simple juxtaposition, soit 20,54 \% de toutes les occurrences des trois tournures, 18 emplois de la tournure avec la préposition $a$ , soit $8,21 \%$ et 156 exemples avec la préposition de, soit 71,23\%. 


\section{1) La juxtaposition ou tournure sans préposition.}

\section{pere ou mere.}

Fors del manoir sa mere issi (80) :c'est ainsi qu'il sortit du manoir de sa mère.

La sont li herceor ma mere (300) :là-bas se trouvent les herseurs de ma mère.

$\mathrm{Au}$ los et au consoil lor pere (460) :sur la recommandation et le conseil de leur père.

Autres occurrences : 727, 1389, 1417.

a) Presque tous les emplois des compléments absolus obéissent aux facteurs que nous avons définis plus haut. En effet, 44 sur les 45 obéissent à ces facteurs. Ces compléments expriment soit un rapport de possession, soit un rapport de parenté. social eleve et il $y$ a absence de déterminants du complement quand celui-ci est un non propre ou Dieu :

Et essillié furent a tort

Li jantil home aprés la mort

Uterpandragon, qui roi fu (443-445)

Les nobles gens furent détruits à tort après la mort d'Uterpandragon qui fut roi.

Que li ange Deu nostre Sire

Sont si tres bel qu'onques Nature

$\mathrm{Ne}$ fist si bele criature (384-386)Que les anges de Dieu Notre-Seigneur sont d'une si

grande beauté que jamais Nature ne fit si belles créatures.

Cf. aussi vers 581, 1008, 1398, 2005, 2370, 4960, 4983, 5230, 5246.

plus souvent déjà cité dans le texte :

Crestiiens, qui antant et painne

A rimoiier le meillor conte

vers le meilleur conte qui soit sur l'ordre du comte.

élevées dans le texte:

andragon, qui rois fu

Vos iroiz a la cort le roi (512):Vous vous rendrez à la cour du roi.

Cf. aussi les vers 971, 1088, 1247, 1368, 2314, 2352, 2694, 4219, 4439, 4934.

possessif accompagne aussi d'autres noms communs appartenant au vocabulaire du monde courtois et de la chevalerie.

...Car le cuer son ami se tout (1032) : car il s'aliène le coeur de son ami.

Qu'il veïst la color novele

De la face s'amie bele (4209-4210)

Qu'il voyait les fraîches couleurs de sa si belle amie.

- Lorsque le complément n'est pas un nom, il est quelquefois un pronom démonstratif suivi d'une relative :

- Uns leus ou an fet le servise

Celui qui ciel et terre fist (574-575)Un lieu où l'on célèbre le service de celui qui créa le

ciel et la terre.

Donez moi le armes celui

Que j'ancontrai defors la porte (998-999)Donnez-moi les armes de celui que j'ai rencontré là-dehors devant la porte. 


$$
\text { autrui : }
$$

Qui changera ne loing ne pres

Ses bons dras por autrui mauvés (1171-1172)Qui échangera d'une manière ou de l'autre

ses bons vêtements à lui contre les mauvais vêtements d'autrui.

Le complément conte fait l'action énoncée par le terme complété comandement et dans l'exemple suivant c'est la reine qui éprouve le sentiment de honte, elle n'en est pas l'objet :

Ne de son duel ne de la honte

La reïne ne li chaut il (970-971)Quant à la douleur et à la honte de la reine, c'est pour lui sans importance.

b) Nous avons cependant trouvé un seul cas d'emploi de la juxtaposition dans lequel le complément est un pronom personnel :

...-Que vos creoiz

Le consoil vostre mere et moi (1416-1417)Que vous croyiez les conseils de votre mère et de moi-même.

Soulignons que cet emploi du pronom personnel en tournure directe est très rare et même exceptionnel. On pourrait l'interpréter comme une volonté de l'auteur d'éviter une double construction qui serait composée de la tournure directe avec le mot mere et de la tournure avec préposition suivie du pronom personnel moi. C'est le seul cas d'emploi de la tournure directe qui n'obéit pas aux facteurs que nous avons définis plus haut.

\section{2) La tournure avec la préposition a}

a) L'emploi de la préposition $\boldsymbol{a}$ dans les domaines réservés à la tournure directe

Nous avons recensé 7 cas d'emplois de la préposition $\boldsymbol{a}$ là où tous les facteurs étaient réunis pour permettre l'emploi du tour non -prépositionnel :

Que changeroie mes bons dras,

Que ma mere me fist l'autr'ier,

Por les dras a cest chevalier? (1162-1164 ) Quoi, j'échangerais mes bons vêtements, que ma mére m'a faits l'autre jour, contre ceux de ce chevalier?

Les armes de sinople taintes

Au chevalier qui hontes maintes

M'a feites selonc son pooir? (1221-1223 )Les armes rouges du chevalier qui m'a fait

toutes les hontes qu'il a pu?

Qui aporta un mantel gris;

Au col au chevalier l'a mis (1779-1780)

Qui apporta un manteau gris qu'il mit au cou du chevalier.

Cf. aussi vers 74, 975, 1598, 2294.

Notons que dans tous ces exemples les compléments désignent des personnes nobles, ils sont déterminés par l'article défini, l'adjectif possessif ou démonstratif. Ils sont tous au singulier et ont une valeur «subjective ». Ces exemples montrent donc que déjà au XII ${ }^{e}$ siècle la préposition avient concurrencer la tournure directe dans son propre domaine.

b) L'emploi de la préposition adans un domaine interdit à la tournure directe

34

Dans l'exemple suivant, le substantif parsome est accompagné d'un article défini auquel il donne sa valeur généralisante :

Le non sachiez a la parsome (561) :finissez par savoir le nom de la personne 
- Le complément, dans les exemples suivants, est un être humain n'appartenant pas à un rang social élevé :

Li vaslez ne prise un denier ${ }^{7}$

Les noveles au charbonier (859-860)

Le jeune homme ne prêta aucun intérêt aux nouvelles du charbonnier.

Et jusqu'a la main au valet

Coloit cele gote vermoille (3200-3201)

Et jusqu'à la main du valet coulait cette goutte vermeille.

La préposition a exprime le plus souvent dans ces exemples un rapport de possession. Dans un seul exemple il marque la parenté : li filz a la veve dame (74),le fils de la veuve.

c) La tournure avec la préposition de

Contrairement à la préposition $a$, la préposition de est très fréquemment employée dans le roman. Et si la préposition a dans la plupart de ses emplois se rencontre dans le domaine propre à la juxtaposition, en revanche la préposition de est plus fréquente dans un tout autre domaine.

d) Emploi de la préposition de dans un domaine réservé à la tournure directe :

Del vaslet qui sa voie tint

Vit les esclos, si li greva (784-785)Il remarqua les traces laissées par le cheval du jeune

homme qui avait poursuivi sa route.

Sanz los d'autrui et sans consoil (2595): sans l'avis ni le conseil de personne.

Remarquons que pour le cas de l'indéfini autrui Chrétien a préféré, dans une autre séquence similaire, employer la tournure directe (cf. vers 460, au los et au consoil lor pere: sur la recommandation et le conseil de leur père) que l'ancien français privilégie surtout quand le complément est le pronom indéfini autrui. Nous pouvons interpréter le choix de Chrétien comme lié à l'ordre des mots, c'est-à-dire que lorsque autrui est employé en tournure directe, il précède le terme complété, comme nous l'avons vu au vers 1172 : ses bons dras por autrui mauvés !, et ici autrui suit le terme complété aussi est-il accompagné de la préposition de.

e) Emploi de la préposition de dans un domaine qui exclut la tournure directe

Dans ces exemples, le complément est l'objet de sentiment indiqué par le terme complété.

Le duel d'un chevalier est le deuil dont le chevalier est l'objet.

- Le complément a une valeur générale, il n'a pas été actualisé :

A la meniere et a la guise

De Galois fu apareilliez (602-603)

Il fut équipé à la mode des Gallois.

Autres occurrences : 934, 1807, 1826, 1939.

Dans ces exemples, le caractère indéterminé du complément est bien marqué par l'absence du déterminant.

- Le complément est un pronom personnel :

Del de moi, quant la leissai,

Cheï pasmee, bien le sai (1587-1588)

C'est du chagrin de me voir partir qu'elle est tombée évanouie.

Qu'ele est morte de duel de toi (3595) :car elle morte de chagrin à cause de toi.

Lorsque le complément est un pronom personnel, on n'utilise presque jamais la tournure directe, mais fait toujours appel à la préposition de.

- Le complément ne désigne pas un animé humain. 
Dans les exemples suivants, le complément est un nom d'animal :

Un petit le fet avant pandre

Tant qu'au col del cheval le joint (1440-1441)

Il le laisse pendre un peu devant de manière à toucher le cou du cheval.

Cf. aussi vers 743, 3271, 3275, 3281, 3404, 4298, 4626, 4627a, 4627b, 5387.

Devant un nom d'animal, de est parfaitement à sa place pour exprimer la possession. Lorsque le complément est un nom de chose, de a une fréquence très élevée dans le texte. La préposition introduit alors plusieurs sortes de rapports.

- Un rapport de possession :

Car sovant hurtoient as armes

Li rain des chasmes et des charmes (105-106)Car souvent se heurtaient aux armes les branches des chênes et des charmes.

Autres occurrences : 110, 212, 621, 640, 669, 740 1326, 1335, 1585, 1778, 2217, 2219, 2297, 2406, 2439, 2449, 2551, 2637a, 2637b, 2689, 2779, 2787, 2823, 2983, 3051, 3162, $3231,3306,3345,3355,3357,3367,4095,4187,4300,4341,4427,4451,4657,4750$, $4897,5019,5116,5206,5269,5498,5572,5663,5685,5704,5849$.

- La préposition introduit aussi des rapports de matière, de partie, de propos, d'origine, d'apposition :

Un bocel trueve plain de vin

Et un henap d'arjant selonc,

Et voit sor un trossel de jonc (738-740)

Il trouva un baril plein de vin et un hanap d'argent à côté, et il vit sur une botte de joncs.

Voir aussi vers 867, 870, 1000, 1079, 1165, 1343, 1352, 1428, 1601, 1603, 1849, 1952, 2033, 2551a, 2551b, 2796, 2803, 2943, 3054, 3074, 3087, 3233, 3567, 4300, 4658, 4751, 5760 .

- Que li autre, se Deus me gart,

N'ont mie de biauté le disme (148-149) Que les autres, Dieu me garde! n'ont pas le dixième de sa beauté.

Cf. aussi vers 202, 2617, 3235a, 3235 b, 5116.

- Ce est li contes del Graal (66) : C'est le conte du Graal

Cf. aussi vers 4566, 5614.

- Que li filz a la veve dame

De la gaste forest soutainne (74-75)

Le fils de la veuve de la solitaire Forêt Déserte

Voir aussi vers 951, 2005, 2776, 3817, 4127.

52 C'est ainsi qu'ont été formés beaucoup de noms propres.

Gornemanz de Goort ai non (1548) : mon nom est Gornemant de Gort.

Voir aussi vers 3817, 3931, 4045, 4825, 4828, 4834, 4835, 4839, 4997, 5003, 5022, 5394, 5409.

- La préposition de introduit un rapport d'apposition :

Con s'il fust jorz d'ascension

Ou autel come au diemoinne,

Qu'alé i furent tuit li moinne (2940-2942)Qu'on se serait cru au jour de l'Ascension ou un dimanche, car tous les moines y étaient venus.

54 Un inventaire des différentes formes de compléments de nom dans le Conte du Graal montre que Chrétien de Troyes fait grand usage de la tournure directe chaque fois que le complément de nom désigne un animé humain au singulier, quand ce complément a une valeur «subjective" et qu'il se trouve bien défini par un déterminant propre à le particulariser. Il arrive toutefois à Chrétien de faire appel à la préposition $a$ alors que tous 
les facteurs permettent l'emploi de la tournure directe ; mais ce n'est pas très courant. En revanche, la préposition de a une fréquence très élevée dans les domaines d'emploi différents de celui de la tournure directe.

Un emploi courant et banal de la préposition de est celui où il précède les noms d'animal ou de chose, et introduit ainsi une foule de rapports différents les uns des autres dont les plus courants sont la matière, la partie, le propos, l'origine.

Notons que les compléments de nom font partie de l'évolution intérieure du personnage dans son itinéraire. Ils appartiennent le plus souvent à trois champs sémantiques. Le premier champ est dominé par la figure maternelle (avec de nombreuses occurrences de "la meison ma mere »), le deuxième champ sémantique est marqué par l'image du monde plus large, plus ouvert à la chevalerie et à la courtoisie (avec les figures du roi, de la reine, des demoiselles d'honneur de la reine, des chevaliers, etc.), le troisième champ sémantique est dominé par la figure de Dieu.

Le Conte du Graal est le roman d'une éducation et les compléments absolus se réfèrent aux trois éducations qui se succèdent dans la vie du héros: la mère, la société noble, chevaleresque et courtoise, Dieu.

La tournure directe, devenue archaïque dès le $\mathrm{XV}^{\mathrm{e}}$ siècle, n'a pas totalement disparu dans la mesure où nous en avons des survivances dans les expressions figées comme Dieu merci, bain-marie, dans de nombreux jurons (ventre Dieu, mort Dieu, où Dieu est généralement déformé en bleu) et de nombreuses désignations de lieu (Vaux-le-Vicomte, Bourg-la-Reine, etc.) et nous restons encore fidèles à l'ancien usage pour désigner les rues, les fêtes et les monuments, du moins quand le complément est un nom de personne, dans les dénominations commerciales, les expressions parlementaires, politiques et juridiques : $\mathrm{La}$ Saint-Jean, rue Monsieur-le-Prince, loi Grammont, affaire Clémenceau.

\section{NOTES}

1. Par le terme dépendance, nous entendons les différentes sortes de rapports (excepté le rapport de parenté et de possession) que le substantif complément entretient avec le terme complété : rapports de partie, d'origine, de genre ou d'espèce, de qualité, de matière, etc.

2. Les inscriptions et les formules constituent des sources d'inspirations pour maints auteurs désireux d'étudier la syntaxe du latin vulgaire.

3. Voir L. Foulet (1963: 14-19).

4. Une thèse a été présentée sur ce tour à la fin du $19^{\mathrm{e}}$ siècle par A. Westholm : Etude historique sur la construction « li filz le rei » en français, Vesteràs, 1899.

5. Nous avons choisi, pour notre étude, la version traduite et présentée par J. Dufournet (1997) d'après la copie de Guiot B. N., f.f. 794.

6. Nous ne pouvons entreprendre cette étude sans noter que deux articles sont publiés sur le sujet dans la revue L'Information grammaticale: A. Queffelec, «La construction sn1 - sn2 et ses concurrentes dans La Mort le roi Artu», 65, mars 1995, pp.12-16 et R. Bellon, « La construction du complément déterminatif dans Le conte du Graal », 81, mars 1999, pp.19-23. 
7. Il convient de faire remarquer que le mot vaslez a deux sens différents dans le texte. Le mot valet est un diminutif du mot vassal. Il vient du latin vassalitus et désigne un jeune noble attaché au service d'un seigneur. Dans ce mot, il y a deux idées: une idée de jeunesse et une idée de service. Par la suite, seule l'idée de service sera développée ; c'est pourquoi le mot valet désigne à partir du XIV ${ }^{\mathrm{e}}$ siècle le serviteur. Dans ce texte de la fin du XII ${ }^{\mathrm{e}}$ siècle, le mot véhicule les deux idées : lorsque l'auteur désigne Perceval par ce mot, il met l'accent sur l'idée de jeunesse et le mot signifie alors « jeune homme noble ", c'est le sens qu'il a aux vers 859 et 784 . Par contre au vers 3200 , c'est l'idée de service qui est mise en relief, c'est pourquoi nous l'avons traduit par valet (serviteur).

\section{AUTEUR}

\section{FIDĖLE DIEDHIOU}

Université Paris 10 - Nanterre 